Phoenix, Arizona USA - December 1999

\title{
Emerging Approaches to Flow Control in Hydrodynamics
}

\author{
Promode R. Bandyopadhyay \\ Naval Undersea Warfare Center \\ Newport, Rhode Island \\ BandyopadhyayPR@Npt.NUWC.Navy.Mil
}

Paper ID CDC99-INV0134

Paper Type: Invited, Regular

Session: Emerging control paradigms in fluid flow control

The $38^{\text {th }}$ IEEE Conference on Decision and Control

Control Systems Society

Dec. 7-10, 1999

Phoenix, AZ 


\title{
Emerging Approaches to Flow Control in Hydrodynamics
}

\author{
Promode R. Bandyopadhyay \\ Naval Undersea Warfare Center \\ Newport, Rhode Island \\ BandyopadhyayPR@Npt.NUWC.Navy.Mil
}

Recent developments in two approaches to hydrodynamic flow control are considered. One is concerned with the closed-loop large-scale control of maneuvering of biologically-inspired small underwater vehicles in a disturbed littoral ocean environment. The other is the small-scale closed- and open-loop control of turbulence in a boundary layer developing on the outer surface of an ocean going vehicle. Theoretical and experimental progress made are described. The former type appears to be well amenable to closed-loop active control with the potential payoff of a high degree of precision in maneuvering. In the latter type, an active open-loop control appears to have the best potential for turbulence quieting and drag reduction. The paper is limited to exploratory work carried out by the author.

\section{Introduction}

The application of control theory to hydrodynamic problems now appears to be promising. This may lead to new and significant performance gains. This is becoming possible because we now have a better knowledge of unsteady vortex hydrodynamics (Fig. 1). This stage has been reached via decades of research. They can be termed as successive periods of focus on steady state hydrodynamics, statistically averaged unsteady hydrodynamics, quasi-deterministic and deterministic hydrodynamics. The realization of the existence of strong identifiable large or small-scale vortex-based hydrodynamics, inherently responsible for the production of forces and moments, has been a crucial development. The successful use of such mechanisms defines the characteristics of many aquatic animals. This also provides a foundation to recent exploration of large-scale active control of underwater bodies. The further realization that,

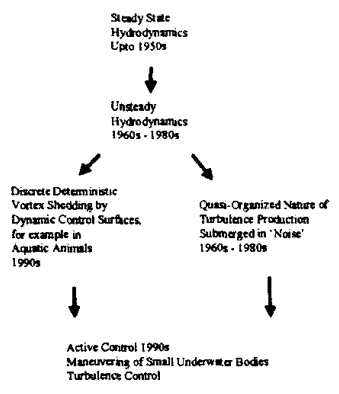

Figure 1. Trends in hydrodynamics research leading to active control

within the seeming randomness of boundary-layer turbulence, actually there are deterministic mechanisms in play although they appear obscured by noise, has opened the door to ideas of small scale control of boundary layer turbulence. In sections 2 and 3 , respectively, we will provide examples of progress made along these two approaches to hydrodynamic control. This summary is limited to the author's experience.

\section{Theoretical Control of Maneuvering of Biologically- Inspired Small Bodies Under Surface Waves}

The littoral region of oceans is highly disturbed due to the presence of surface waves, a sea floor, cross-currents and submerged obstacles. Much can be learned about maneuvering in such environment by studying the fin motions of fish. After studying the fin morphology of many species of fish, classified as slow but highly maneuverable, fast but poorly maneuverable, and an overlapping class, namely fast yet highly maneuverable, it was found that all species endowed with maneuverability have a well defined fin morphology (Ref. 3). The dynamics of dorsal (longitudinal ridge) and caudal (tail) fins, as well as of head swaying, have been simulated on rigid cylinders in water tunnels and tow tanks (Ref. 5). The work of Profs. Triantafyllou at MIT and CCNY (Ref. 15) has placed the discrete vortices formed by the tail motion of a fish and their Strouhal number $(S t=f A / U$, where $f$ is tail flapping frequency, $A$ is maximum tail displacement and $U$ is speed of fish) squarely at the center of the debate on the propulsive capability of a fish. Dynamic force measurements due to flapping foils attached to a rigid cylinder indicate that the levels of instantaneous and time averaged forces on the cylinder can be 100 and 50 times of the steady state drag levels, respectively (Ref. 5). These results suggest that the dynamic flapping motions utilized by aquatic animals have an intrinsic amenability to active control - a feature that is not currently utilized in current underwater vehicles. For example, a system of discrete vortices can be produced on a main body by means of several dynamic control surfaces, and then their phase relationships can be tuned to produce a desired precision maneuvering. In the following, an example of such a control of maneuvering of a small cylinder that is in motion near surface waves is described.

2.1 Problem: A control system is being designed for low-speed maneuvering of small undersea vehicles using dorsal- and caudal-like fins (Fig. 2). The details are given in Ref. 7. A hydrodynamic control scheme is developed so that the vehicle tracks a precise depth versus time trajectory. It is assumed that the hydrodynamic parameters of the vehicle are imprecisely known and surface waveinduced forces are constantly acting on the vehicle. 
Although the design approach can be extended to yaw control, in this study, only control in the dive plane is considered. Using the dorsal fin, a normal force is produced for depth control and flapping foils produce pitching moment for pitch angle regulation. For simplicity, it is assumed that the vehicle is equipped with a control mechanism that causes the vehicle to move forward with a uniform velocity. For the depth trajectory control, an adaptive sliding mode control law is designed for the continuous cambering of the dorsal fins in the presence of sea waves. The sliding mode control law is nonlinear and discontinuous in the state space and has an excellent insensitivity property with respect to disturbances and parameter variations.

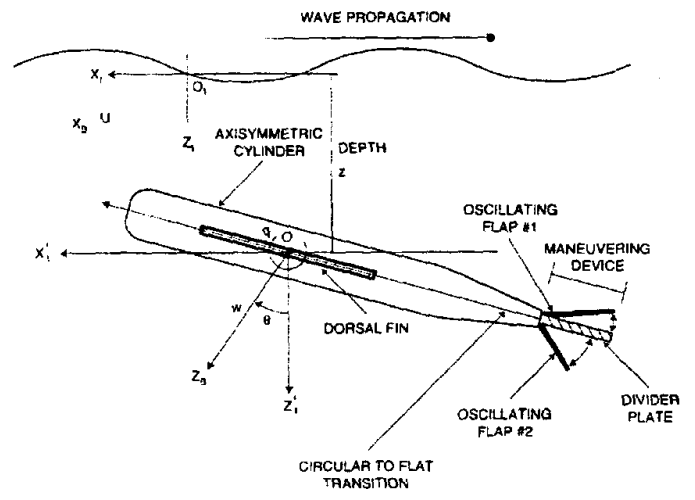

Figure 2. Schematic of the Maneuvering Devices (Dorsal and Caudal Fins) and Axisymmetric Cylinder. Note that the long dorsal fins are actually mounted in the horizontal plane. The caudal fins are also mounted in the horizontal plane and are akin to flukes in whales.

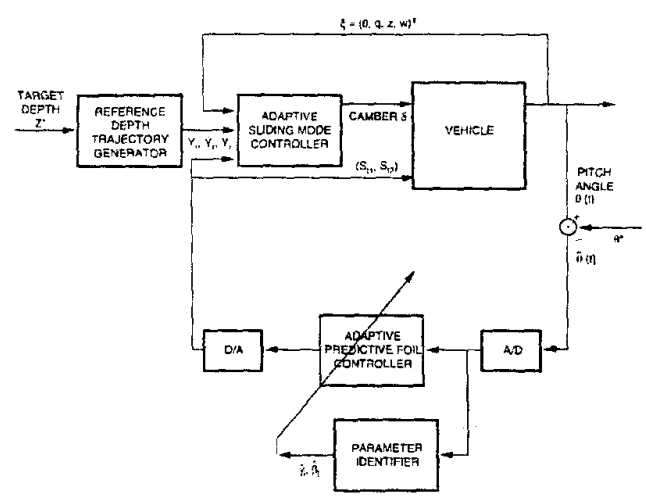

Figure 3. Closed-Loop System (Including the Caudal and Dorsal Fin Controllers)

2.2 Methodology: The heave and pitch equations of motion are described by coupled nonlinear differential equations. The closed loop system is shown schematically in Fig. 3.

2.3 Results: The experimental vehicle data from Ref. 6 was used to carryout a SIMULINK simulation. A typical result of depth tracking is shown in Fig. 4.
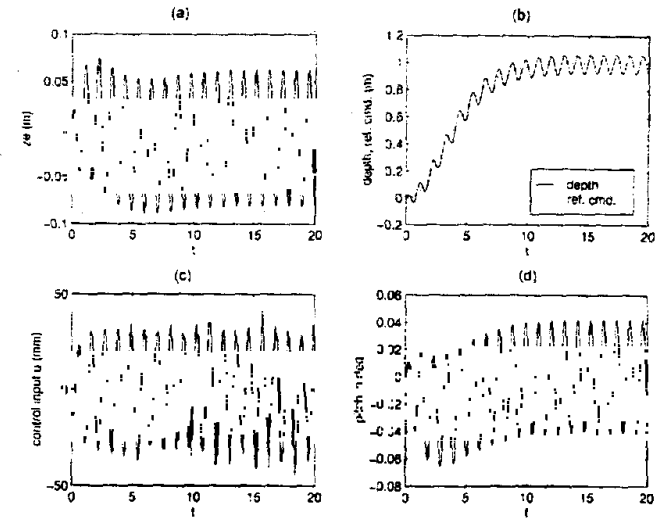

Figure 4. Dorsal Fin Control: Sinusoidal Disturbance: (a) Depth error $z_{e}$ $=z-y_{r}$ (b) Depth $z$ and reference command $y_{n}$ (c) Camber $\delta=u_{i}(d)$

$$
\text { Pitch angle }
$$

\section{Biomimetics}

The successful fabrication of active maneuvering concepts shown in the previous section depends on the actuator technology. Electrocapacitive hydrophilic platinum composite polymer fins, also called biomimetic muscles, are being developed as such actuators. Motors, gears and shafts are absent, leading to a saving in space, and they perform quietly. Currently, stacks of such layers $(0.2 \mathrm{~mm}$ thick) operate at less than $10 \mathrm{v}$ and at frequencies $0.5-5 \mathrm{~Hz}$. Deflections of $10 \mathrm{~mm}$ can be produced in a fin length of 30 $\mathrm{mm}$. The electrodes are integrated with hydrofoils (see video Ref. 9). Figure 5 shows frames of video showing deflected fins in water.

(al)

(b1)
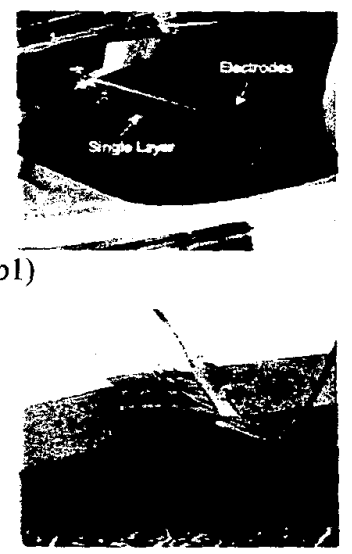

(c1)

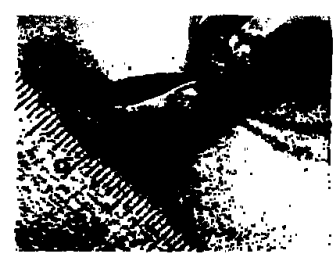

Figure S. Biomimetic fin actuators in water. Flap on hydrofoil (label: Electrodes \& Single Layer): (a1) closed; (a2) open. Oppositely oscillating (a2)

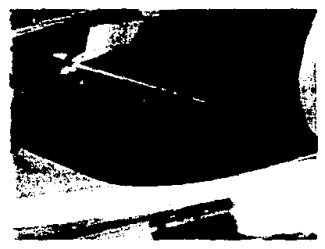

(b2)

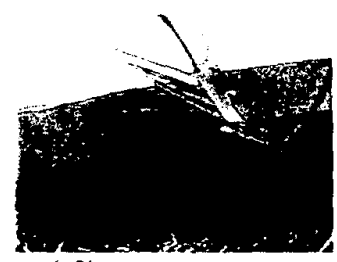

(c2)

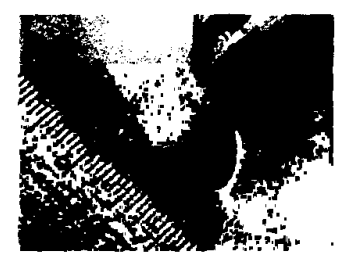


rows of comb vortex generators on a hydrofoil: (b1 \& b2), and hydrofoil tail flap deflected and held to left (c1) and to right (c2). Ruler is in inches; maximum deflection is $35 \mathrm{~mm}$.

\section{Control of Boundary-Layer Turbulence}

The mechanism of turbulence production in a turbulent boundary layer developing on a vehicle skin is only partly deterministic. They have a wide standard of deviation in spatial and temporal scales and in their place of occurrence. Due to this reason, an instantaneous or individual vortex based control, as in the previous example, is a nightmare. It is more so at high Reynolds number where the scales become smaller and they abound in number. In the following, an example of a closed-loop control is given. This is followed by open loop approaches, which perhaps has a greater potential for success.

4.1 Problem: The control of saltwater turbulence using electromagnetic actuators is considered. First, the theoretical closed-loop control of (uncoupled) perturbations is considered. Then, the progress made in the development of actuators is considered. Next, a physical hydrodynamic model is presented for open loop control to achieve suppression of drag and turbulence. Finally, an open-loop MEMS actuator is presented for suppression of wallpressure fluctuations

\subsection{Theoretical Linear Feedback Control Using Electromagnetic Actuators}

The feasibility of a closed-loop control of the fluctuations in a two-dimensional turbulent boundary layer is explored (Ref. 14).

4.2.1 Methodology: The fluid medium is salt water. The actuator consists of small surface mounted magnets and electrodes, which produce a Lorenz force field. A system theory approach is taken. Mean and turbulence are assumed to be decoupled. Beginning with the two-dimensional Navier-Stokes equations of motion, a finite, dimensional, linear state variable, approximate model is obtained using Galerkin's procedure. Based on this model, linear feedback control laws are obtained to achieve stabilization of the perturbed flow to the base flow. Figure 6 shows the closedloop system, including the optimal controller (solid line) and the simple controller using wall-shear stress feedback (dotted line).

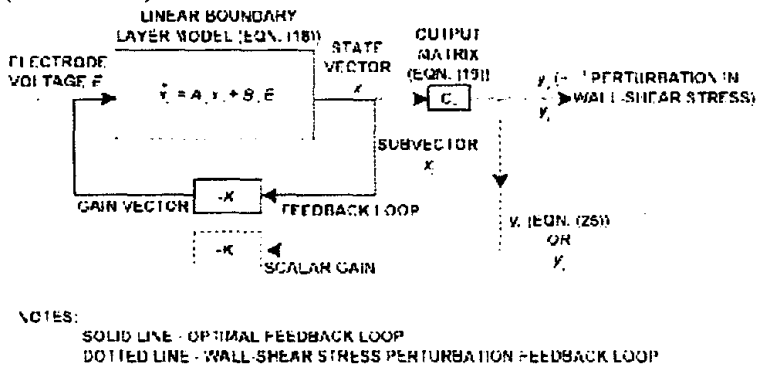

Figure 6. Closed-Loop Lorenz Force Control System

4.2.2 Results: It is shown that spatially distributed longitudinal or surface-normal forces stabilize the force perturbations. However, for lower wave numbers, longitudinal forces are more effective because surfacenormal forces require larger electrode voltages for the same response characteristics. Simulated results are presented in Figs. 7 and 8 to show how stabilization is achieved in the closed loop system.
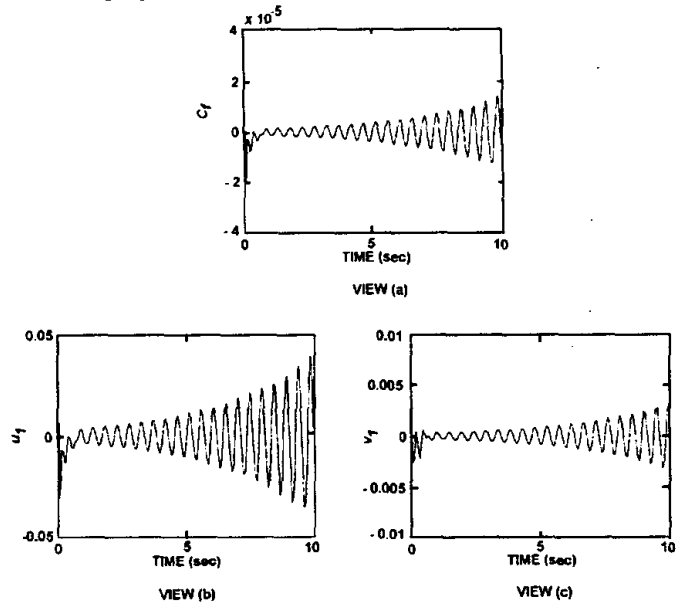

Figure 7. Uncontrolled System at $x^{*}=0.751_{1}, y^{*}=-0.409$ Above the Plate
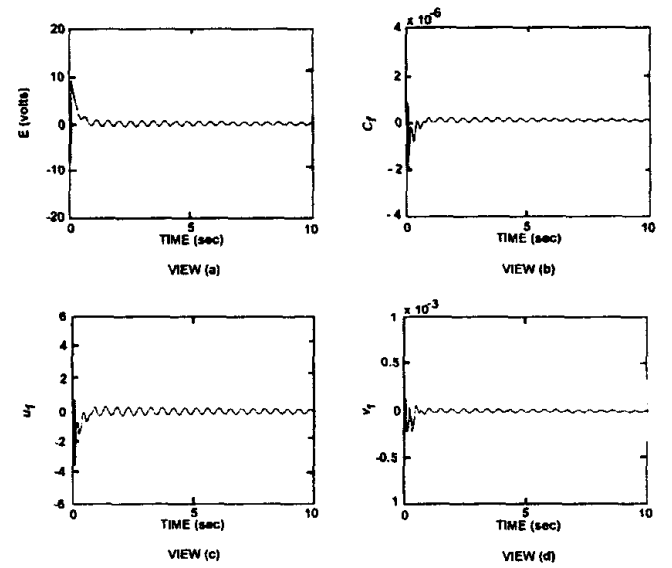

Figure 8. Longitudinal force control by wall-shear stress feedback at location in Fig. 7.

\subsection{Open Loop Active Control Using Electromagnetic Actuators}

Drag reduction and turbulence control is an extremely difficult problem. Utilization of Lorenz force to pump near wall saltwater in a turbulent boundary layer developing on an ocean going hull has many appeals. An open-loop active control working on a well-founded hydrodynamic principle, that does not address the control of each individual vortex, is more likely to succeed. The DNS drag reduction demonstration of Akhavan (Ref. 12) and the experimental verification by Choi et al. (Ref. 10) and Laadhari et al. (Ref. 13) are used to develop such a hydrodynamic model of drag reduction in Ref. 1. 
4.3.1 Methodologl: This is a kinematic model and is shown in Fig. 9. The reduction of drag due to turbulence is under consideration. When the normal streamwise vortices $\left(\omega_{r}\right)$ are reoriented along span. say via spanwise oscillation as in a Stokes layer, drag is reduced as per the kinematic sinusoidal relationship given in Fig. 9 .

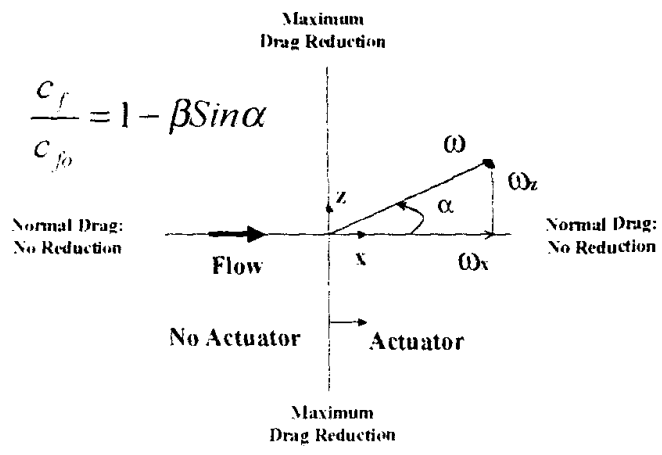

Figure 9. Vorticity reorientation model of drag reduction due to the present author (Ref. I).

\subsubsection{Results:}

The visualization of Choi et al.'s drag reduction experiment was examined for evidence of the vorticity reorientation model in Fig. 9. An example is shown in Fig. 10.

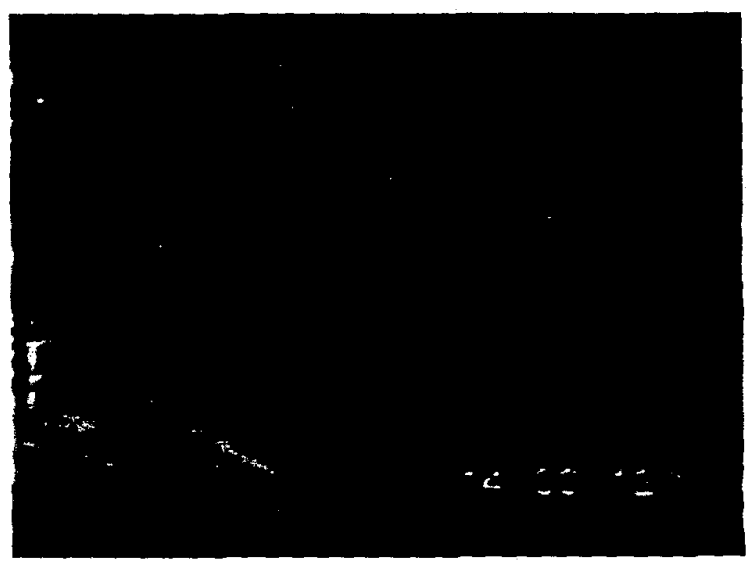

Figure 10. Visualization of inclined vorticity waves by spanwise wall oscillation. Extracted from video by Choi at al.

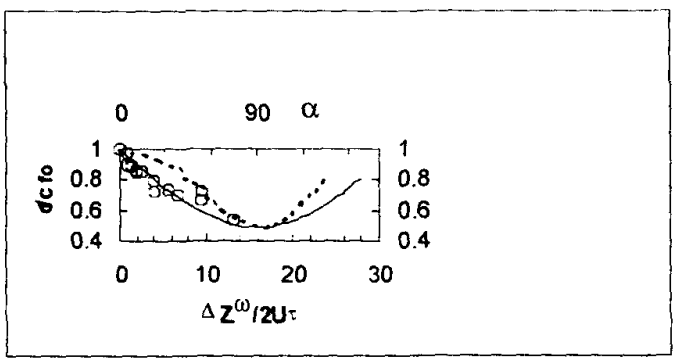

Figure 11. Comparison of vorticity reorientation model (solid line) with Akhavan's Direct Numerical Simulation (1992) of Navier-Stokes equations (broken line) and measurements of Choi et al. (1998) and Laadhari et al. (1994).

It has been shown in Ref. 1 that Stokes waves are formed in a normal turbulent boundary layer when the near wall fluid is sloshed along span. These Stokes waves are skewed normal to the wall and are attenuated compared to those in a classical no-mean-flow laminar environment. This model leads to a remarkable agreement with measurements (Fig 11). The vorticity reorientation model in Fig. 9 is a recent development and an actuator that can produce the spanwise near wall sloshing, given in Fig. 11 is being built. Figure 12 shows our earlier effort on actuator design (Refs. $2,4 \& 11$ )

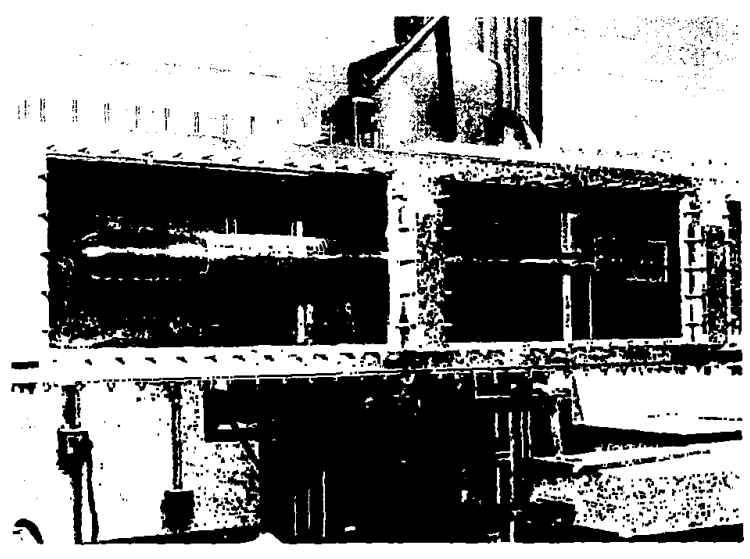

Figure 12. Photograph of a $75 \mathrm{~mm}$ diameter cylinder model containing numerous surface mounted electromagnetic actuators to pump saltwater near wall by means of Lorenz forces. The model is mounted in a saltwater tunnel.

\subsection{Open Loop Passive Control of Wall-Pressure}

\section{Fluctuations Using MEMS Actuators}

One of the sources of radiated noise in underwater vehicles is the wall-pressure fluctuations in turbulent boundary layer developing over hulls. The spatial and temporal locations of these fluctuations are quasi-deterministic.

4.4.1 Methodology: This works on the principle that surface tension forces are high at micron levels and can be used as force dampers (Ref. 8). An approach amenable to active control has been taken. A MEMS fabricated surface has been tested in a quiet water tunnel. The surface consists of

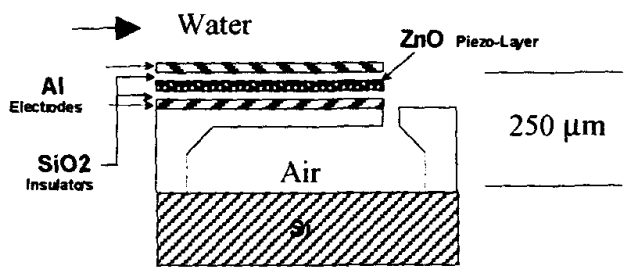

Figure 13. Cross-section of one cavity with an active lid.

numerous micro-cavities each with a flexible diving board lid on top (Figs. $13 \& 14$ ). Their size, spacing and temporal scales are scaled to boundary layer turbulence Reynolds number. The cavities have a tiny pocket of air, while the 
hull is submerged in water. Thus, each cavity-lid combination is like a little 'air bed', in analogy to popular 'water beds'.

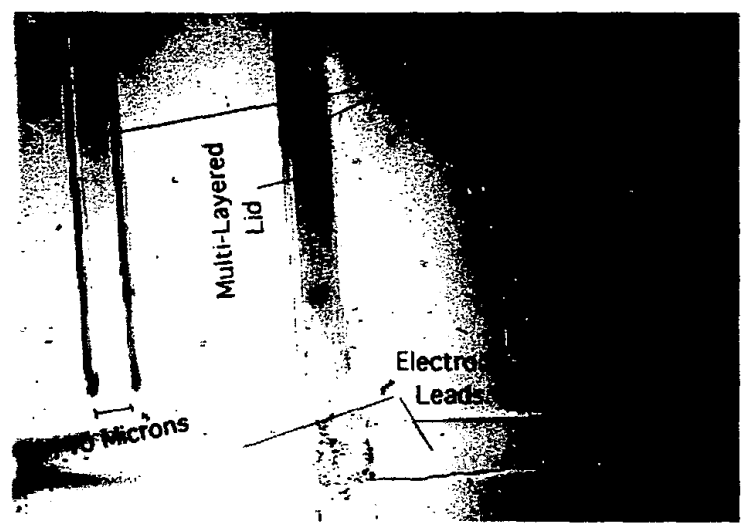

Figure 14. Photograph of the Silicon elastic Lid (10 $\mu \mathrm{m}$ thick) After Multi-Layering Shown in Fig. 13.

4.4.2 Results: The details are given in Ref. 10. In an exploratory experiment at high Reynolds number, in the passive mode, a marginal reduction in the wall-pressure spectrum was achieved even after an averaging of 500 realizations. Work is continuing on simulation and optimization.

\section{Conclusions}

In hydrodynamics, the realization that discrete deterministic or quasi-deterministic vortex shedding is involved in many practical flows in what previously was thought to be random, has given an impetus to the application of active control methodologies to unsteady hydrodynamics. New applications in maneuverability and turbulence control are being explored now which were not feasible before. Some of the developments in actuator technologies, namely biomimetics, MEMS and micro-electromagnetics have been summarized. Several laboratory and simulation results have been given which demonstrate the challenges and potential.

\section{Acknowledgment}

Work supported by the Office of Naval Research (Program Managers: Dr. Teresa McMullen, James Fein and Dr. L. Patrick Purtell) and NUWC IR (Dr. Stuart Dickinson). Their interest and encouragement are deeply acknowledged. The support of Prof. S. Singh, Drs. M. Mojarrad, M. Donnelly, Messrs. J. Castano, W. Nedderman, D. Thivierge and J. Raposa are acknowledged. Discussions with Prof. P. S. Virk on Stokes mechanism led to improvements.

\section{References}

1. Bandyopadhyay, P. R. 1999 “The Stokes' Mechanism of Drag Reduction," (in Preparation).

2. Bandyopadhyay, P. R. \& Castano, J. M. 1996 "Microtiles for Electromagnetic Turbulence Control in Seawater - Preliminary Experiments," ASME FED Vol. 237, Vol. 2, pp. 53-60, ASME Symposium on Drag Reduction, July 1996, San Diego.
3. Bandyopadhyay, P. R., Castano, J. M., Rice, J. Q. Philips, R. B., Nedderman, W. H. \& Macy, W. K. 1997 "Low-speed Maneuvering Hydrodynamics of Fish and Small Underwater Vehicles" ASME Jou. Fluids Engrg., V119, pp. 136-144.

4. Bandyopadhyay, P. R., Castano, J., Thivierge, D. \& Nedderman, W. H. 1998 "Drag Reduction Experiments on a Small Axisymmetric Body in Saltwater Using Electromagnetic Microtiles", Proc. International Symposium on Seawater Drag Reduction, July 22-24, 1998, NUWC, Newport, RI, pp. 373-378.

5. Bandyopadhyay, P. R. \& Donnelly, M. J. 1999a "Experimental Simulation of Fish-Inspired Unsteady Vortex Dynamics on a Rigid Cylinder," ASME Jou. Fluids Engrg., (subjudice).

6. Bandyopadhyay, P. R., Nedderman, W. H. \& Dick, J. 1999 b "Biologically-Inspired Bodies Under Surface Waves. Part 1: Load Measurements", ASME Jou. Fluids Engrg., Vol. 121, No. 2, pp. 469-478.

7. Bandyopadhyay, P. R., Singh, S. \& Chockalingam, F. 1999c "Biologically-Inspired Bodies Under Surface Waves. Part 2: Theoretical Control of Maneuvering", ASME Jou. Fluids Engrg., Vol. 121, No. 2, pp. 479487.

8. Bandyopadhyay, P. R. 1995 "Microfabricated Silicon Surfaces for Turbulence Control," Proc. of the ACTIVE 95, The International Symposium on Active Control of Sound and Vibration, Newport Beach, CA, July 6-8, 1995, Paper Number 013, pp. 1327-1338.

9. Bandyopadhyay, P. R., Nedderman, W. H., Raposa, J. \& Mojarrad, M. 1999 "Biomimetics: 1999", Edited and Captioned Video, 3:20 Mts.

10. Choi, K.-S., DeBisschop, J.-R. \& Clayton, B. R. 1998 "Turbulent Boundary-Layer Control by Means of Spanwise-Wall Oscillation," AIAA Jou., Vol. 36, 1157-1163.

11. Hatay, F., O' Sullivan, P. L., Biringen, S. \& Bandyopadhyay, P. R. 1997 "Numerical Simulation of Secondary Flows in Channels Driven by Applied Lorentz Forces," AIAA Jou. Thermophysics \& Heat Transf. Vol. 11, No. 3, pp. 446-453.

12. Jung, W. J., Mangiavacchi, N. \& Akhavan, R. 1992 "Suppression of Turbulence in Wall-Bounded Flows by High Frequency Spanwise Oscillations," Phys. Fl., Vol. A4, 1605-1607.

13. Laadhari, F., Skandaji, L. \& Morel, R. 1994

"Turbulence Reduction in a Boundary Layer by a Local Spanwise Oscillating Surface," Phys. Fl., Vol. A6, 3218-3220.

14. Singh, S. N. \& Bandyopadhyay, P. R. 1997 "Linear Feedback Control of Boundary Layer Using Electromagnetic Microtiles," ASME Jou. Fluids Engrg., V119, No. 4, pp. 852-858.

15. Triantafyllou, M. S. \& Triantafyllou, G. S. 1995 "An Efficient Swimming Machine," Scientific American, Vol. 272, 64-70. 